\title{
On singular and highly oscillatory properties of the Green function for ship motions
}

\author{
By XIAO-BO CHEN ${ }^{1}$ AND GUO XIONG WU ${ }^{2}$ \\ ${ }^{1}$ DTA, Bureau Veritas, 17bis, Place des Reflets 92400 Courbevoie, France \\ e-mail: xiao-bo.chen@bureauveritas.com \\ ${ }^{2}$ Department of Mechanical Engineering, University College London, \\ Torrington Place, London WC1E 7JE, UK \\ e-mail: gx_wu@meng.ucl.ac.uk
}

(Received 12 October 2000 and in revised form 11 April 2001)

The Green function used for analysing ship motions in waves is the velocity potential due to a point source pulsating and advancing at a uniform forward speed. The behaviour of this function is investigated, in particular for the case when the source is located at or close to the free surface. In the far field, the Green function is represented by a single integral along one closed dispersion curve and two open dispersion curves. The single integral along the open dispersion curves is analysed based on the asymptotic expansion of a complex error function. The singular and highly oscillatory behaviour of the Green function is captured, which shows that the Green function oscillates with indefinitely increasing amplitude and indefinitely decreasing wavelength, when a field point approaches the track of the source point at the free surface. This sheds some light on the nature of the difficulties in the numerical methods used for predicting the motion of a ship advancing in waves.

\section{Introduction}

The potential flow generated by a source pulsating sinusoidally and moving with constant horizontal velocity is fundamental to the analysis of the flow past a moving ship and to the prediction of its motions in waves. The mathematical solution associated with the linearized free-surface boundary condition, or the so-called shipmotion Green function can be expressed as the sum of a simple (Rankine) source, a double non-oscillatory Fourier integral which is significant only in the near field and a single integral which is dominant in the far field. The second and third components account for the free-surface effects. In particular, the last component has been a major obstacle to the numerical solution of the ship-motion problem. The present work aims to reveal some additional insight into the difficulty.

A special case is the Neumann-Kelvin steady flow problem where the source moves forward only and has no pulsation. Based on the stationary phase method, it can be shown that the single integral at the far field gives the well-known Kelvin wave pattern confined inside the wedge bounded by two critical lines forming an angle of $38^{\circ} 56^{\prime} 32^{\prime \prime}$ (see, for example, Lamb 1932). A similar case is that due to a moving concentrated pressure. It was shown by Ursell (1960) that near the track of the pressure point applied on the free surface, the wave elevation from the linearized theory oscillates with indefinitely increasing amplitude and indefinitely decreasing wavelength. This important result was later analysed in more detail by Euvrard (1983) and Ursell 
(1988). Some aspects of the numerical computation of this complicated function were discussed by Clarisse \& Newman (1994).

For the more general case of a point source both pulsating and advancing at a uniform speed, similar behaviour can be expected when the source is located on the free surface. Understanding of this behaviour is essential if the insight into the physics of ship waves is to be improved. It is also crucially important in the development of the computation code for predicting the ship motion in waves. Here we shall focus our analysis on the behaviour of the wave near the track of the source. For the steady case, Ursell (1960) used Peters' expression (Peters 1949) for the wave field and obtained the result by deforming the path of integration. Here the expression for the wave field is very different from Peters'. A different approach is therefore used, which is outlined below.

Noblesse \& Chen (1995) decomposed the free-surface effect into a local nonoscillatory component and a wave component. The wave component is expressed as a single integral along the dispersion curves defined by the dispersion relation associated with the linear free-surface boundary condition. The dispersion relation usually defines three dispersion curves which can be classified as two open curves along which the wavenumber is not bounded and a closed one along which the wavenumber is finite. The integral then consists of three parts corresponding to three dispersion curves: two indefinite ones with their limits tending to positive and negative infinity respectively, and one definite integral. The last one exists only when $\tau=U \omega / g<1 / 4$, where $U$ is the forward speed, $\omega$ the encounter frequency and $g$ the acceleration due to gravity. Although the integrand of the single integral is not singular, care is needed for the two indefinite integrals, as the unbounded limit can lead to highly oscillatory and singular behaviour. Indeed, the analysis by Chen \& Noblesse (1997) revealed a direct relationship between the geometrical properties of a dispersion curve and important aspects of the corresponding far-field waves, including wavelength, directions of wave propagation, phase and group velocities, and cusp angles. Their work has established the link between the highly-oscillatory and singular properties of the wave component and the open dispersion curves along which two indefinite integrals are performed.

The analysis here is carried out through the leading terms of the wave component associated with the open dispersion curves. The first leading term of the integrand has a non-decaying amplitude while the second leading term is proportional to $1 / a$ where $a$ is the integral variable. The difference between the original integrand and these two leading terms is of order $1 / a^{2}$ when $|a|$ tends to infinity and the result is finite. This means that the behaviour of a wave component can be analysed through the two leading terms.

By making use of asymptotic properties of the complex error function, it is shown that the Green function is highly oscillatory with indefinitely increasing amplitude and indefinitely decreasing wavelength, when the field point approaches the track of the source point located at the free surface. This peculiar behaviour of the ship-motion Green function is demonstrated by numerical results in graphic form. Furthermore, it is noted that when $\omega=0$ the result obtained here are consistent with that found by Ursell $(1960,1988)$ for the steady Neumann-Kelvin problem.

\section{Wave component and open dispersion curves}

A moving Cartesian system $O x y z$ is defined in which $x$ points in the direction of forward speed and $z$ upwards. The origin is located on the undisturbed free surface. 
The coordinates $(x, y, z)$ are non-dimensionalized using a characteristic length $L$ which for a real ship problem is usually chosen as its length. The ship-motion Green function $G(\boldsymbol{\xi}, \boldsymbol{x})$ is defined through $\phi=\operatorname{Re}\left\{G \mathrm{e}^{-\mathrm{i} \omega t}\right\}$ where $\phi$ is the velocity potential at a point $\boldsymbol{\xi}=(\xi, \eta, \zeta)$ generated by a source of unit strength located at a point $\boldsymbol{x}=(x, y, z)$ pulsating sinusoidally with frequency $\omega$ and advancing at constant speed $U$ with the coordinate system. This is a classical problem and details can be found in Wehausen $\&$ Laitone (1960). Here we may write

$$
G=G^{S}+G^{F} .
$$

$G^{S}$ is defined in terms of simple (Rankine) source

$$
4 \pi G^{S}=-1 / r+1 / r^{\prime}
$$

where $r$ is the distance between $\boldsymbol{\xi}$ and $\boldsymbol{x}$, and $r^{\prime}$ the distance between $\boldsymbol{\xi}$ and the mirror image of $\boldsymbol{x}$ with respect to the mean free-surface plane $z=0$. The component $G^{F}$ in equation (1) is introduced to satisfy the linear free-surface boundary condition

$$
\lim _{\epsilon \rightarrow+0}\left[(f+\mathrm{i} \epsilon-\mathrm{i} F \partial / \partial x)^{2}-\partial / \partial z\right] G=0
$$

where $f=\omega \sqrt{L / g}$ and $F=U / \sqrt{g L}$. The role of $\epsilon$ in the above equation is to ensure that the radiation condition is satisfied at the far field, in a manner described by Lighthill (1978, p. 364).

If we use

$$
\begin{gathered}
1 / r=1 /\left(4 \pi^{2}\right) \int_{-\infty}^{\infty} \mathrm{d} \beta \int_{-\infty}^{\infty} \mathrm{d} \alpha \mathrm{e}^{k|\zeta-z|-\mathrm{i}[\alpha(\xi-x)+\beta(\eta-y)]} / k, \\
1 / r^{\prime}=1 /\left(4 \pi^{2}\right) \int_{-\infty}^{\infty} \mathrm{d} \beta \int_{-\infty}^{\infty} \mathrm{d} \alpha \mathrm{e}^{k(\zeta+z)-\mathrm{i}[\alpha(\xi-x)+\beta(\eta-y)]} / k,
\end{gathered}
$$

with $k=\sqrt{\alpha^{2}+\beta^{2}}$, it is straightforward to obtain (Noblesse \& Yang 1995)

$$
4 \pi^{2} G^{F}=\lim _{\epsilon \rightarrow+0} \int_{-\infty}^{\infty} \mathrm{d} \beta \int_{-\infty}^{\infty} \mathrm{d} \alpha \frac{\mathrm{e}^{k(\zeta+z)-\mathrm{i}[\alpha(\xi-x)+\beta(\eta-y)]}}{D+\mathrm{i} \epsilon \operatorname{sign}\left(D_{f}\right)}
$$

where $D$ is the dispersion function

$$
D=(f-F \alpha)^{2}-k
$$

The function $\operatorname{sign}\left(D_{f}\right)$ is given by

$$
\operatorname{sign}\left(D_{f}\right)=\operatorname{sign}(\partial D / \partial f)=\operatorname{sign}(f-F \alpha) .
$$

Equation (5) is written in terms of the Cartesian variables $(\alpha, \beta)$ while the classic result in Wehausen $\&$ Laitone (1960) is given in the polar variables $(k, \theta)$ with $\alpha=k \cos \theta$ and $\beta=k \sin \theta$. In other words, they are the same equations written in a different manner.

Following the analysis of Noblesse \& Chen (1995), equation (5) can be decomposed as $G^{F}=G^{W}+G^{N}$ with $G^{W}$ being the wave component and $G^{N}$ the non-oscillatory local component. As $G^{F} \approx G^{W}$ in the far field, only the wave component $G^{W}$ is considered below. Along the dispersion curves defined by $D=0, G^{W}$ can be written as

$$
4 \pi G^{W}=-\mathrm{i} \sum_{D=0} \int_{D=0} \mathrm{~d} s\left(\Sigma_{1}+\Sigma_{2}\right) \mathrm{e}^{k(\zeta+z)-\mathrm{i}[\alpha(\xi-x)+\beta(\eta-y)]} \mathrm{d} s /\|\nabla D\|
$$


where $\sum_{D=0}$ means summation over all the dispersion curves and $\|\nabla D\|^{2}=D_{\alpha}^{2}+D_{\beta}^{2}$. The function $\Sigma_{1}=\operatorname{sign}\left(D_{f}\right)$ is associated with the limit $\epsilon \rightarrow+0$ in (5) and $\Sigma_{2}$ is given as, following Noblesse \& Chen (1995),

$$
\Sigma_{2}=\operatorname{sign}\left[(\xi-x) D_{\alpha}+(\eta-y) D_{\beta}\right]
$$

The dispersion curves defined, in the plane $(\alpha, \beta)$, by the dispersion relation $D=0$, or

$$
(f-F \alpha)^{2}-k=0
$$

are symmetric with respect to $\beta=0$ so that only that in the upper half-plane given by

$$
\beta=\sqrt{(F \alpha-f)^{4}-\alpha^{2}}
$$

is considered below.

It can be shown based on equation (10) that three or two dispersion curves exist depending on whether $\tau=f F=\omega U / g$ is smaller or larger than $1 / 4$, respectively. For $\tau<1 / 4$, the three dispersion curves intersect the axis $\beta=0$ at four values of $\alpha$, which are denoted $\alpha_{i}^{ \pm}$and $\alpha_{0}^{ \pm}$, and given by

$$
\begin{aligned}
& F^{2} \alpha_{i}^{ \pm}=\tau \pm(1 / 2-\sqrt{1 / 4 \pm \tau}), \\
& F^{2} \alpha_{o}^{ \pm}=\tau \pm(1 / 2+\sqrt{1 / 4 \pm \tau}) .
\end{aligned}
$$

Two open dispersion curves are located in the regions $-\infty<\alpha \leqslant \alpha_{o}^{-}$and $\alpha_{o}^{+} \leqslant \alpha<\infty$, and an inner closed dispersion curve in the region $\alpha_{i}^{-} \leqslant \alpha \leqslant \alpha_{i}^{+}$. For $\tau>1 / 4$, only two open dispersion curves exist and are located in the regions $-\infty<\alpha \leqslant \alpha_{i}^{+}$and $\alpha_{o}^{+} \leqslant \alpha<\infty$.

The main interest here is in the integral along the open dispersion curves, as they are the reason for the singular and highly oscillatory behaviour. To analyse these integrals, it is useful to adopt the Fourier variables scaled by $F^{2}$ so that equation (10) can be written as

$$
F^{2} \beta=\sqrt{\left(F^{2} \alpha-\tau\right)^{4}-\left(F^{2} \alpha\right)^{2}} .
$$

If we further define

$$
(a, b)=\left(F^{2} \alpha-\tau, F^{2} \beta\right)
$$

and $c=F^{2} k$, the dispersion relation in equation (9) can be written

$$
c=F^{2} k=\sqrt{(a+\tau)^{2}+b^{2}}=a^{2} .
$$

Based on the definitions of the new variables, equation (10) can now be written

$$
b=\sqrt{a^{4}-a^{2}-2 \tau a-\tau^{2}} .
$$

The two open dispersion curves defined by this equation are located in $-\infty<a \leqslant a^{-}$ and $a^{+} \leqslant a<\infty$ where

$$
a^{-}=\left\{\begin{array}{lll}
-\sqrt{1 / 4-\tau}-1 / 2 & \text { if } & \tau \leqslant 1 / 4 \\
-\sqrt{1 / 4+\tau}+1 / 2 & \text { if } & \tau>1 / 4
\end{array} \quad \text { and } a^{+}=\sqrt{1 / 4+\tau}+1 / 2\right.
$$

It can be easily verified that $a^{-} \leqslant-(\sqrt{2}-1) / 2$ and $a^{+} \geqslant 1$. At $\tau=0$, the open dispersion curves are symmetrical with respect to $a=0$ and $-a^{-}=1=a^{+}$. 


\section{Analysis of the singular and highly oscillatory wave component}

In (7), $\Sigma_{1}=\operatorname{sign}(-a)= \pm 1$ for the left $\left(a \leqslant a^{-}\right)$and right $\left(a \geqslant a^{+}\right)$dispersion curves, respectively. In (8) for $\Sigma_{2}$, it can be verified that $\operatorname{sign}\left(D_{\alpha}\right)=\operatorname{sign}\left(D_{a}\right)=\mp 1$ for $a \leqslant a^{-}$and $a \geqslant a^{+}$respectively and $D_{\beta}$ is finite. Since only the downstream wave component $(\xi<x)$ and near the track of the source $(\eta-y) \rightarrow 0$, is considered, we have

$$
\Sigma_{2}=\left\{\begin{array}{lll}
+1 & \text { for } \quad a \leqslant a^{-} \\
-1 & \text { for } \quad a \geqslant a^{+}
\end{array}\right.
$$

Along the dispersion curve, $D=0$ gives $D_{\alpha}+D_{\beta}(\mathrm{d} \beta / \mathrm{d} \alpha)=0$. Thus

$$
\mathrm{d} s /\|\nabla D\|=\sqrt{(\mathrm{d} \alpha)^{2}+(\mathrm{d} \beta)^{2}} / \sqrt{D_{\alpha}^{2}+D_{\beta}^{2}}=\mathrm{d} \alpha /\left|D_{\beta}\right|=\mathrm{d} \alpha(k / \beta)=F^{-2} \mathrm{~d} a(c / b) .
$$

Then the wave component associated with the two open dispersion curves becomes

$$
2 \pi F^{2} G^{W}=-\mathrm{i}\left(\int_{-\infty}^{a^{-}}-\int_{a^{+}}^{\infty}\right) \frac{c}{b}\left(\mathscr{E}^{+}+\mathscr{E}^{-}\right) \mathrm{d} a
$$

where $\mathscr{E}^{ \pm}$are given by

$$
\mathscr{E}^{ \pm}=\mathrm{e}^{k(\zeta+z)-\mathrm{i}[\alpha(\xi-x) \pm \beta(\eta-y)]}=\exp \left[Z c-\mathrm{i} X(a+\tau)-\mathrm{i} Y^{ \pm} b\right],
$$

with

$$
(X, Y, Z)=(\xi-x, \eta-y, \zeta+z) / F^{2}, \quad X<0, \quad Y^{ \pm}= \pm Y
$$

and $Y \geqslant 0$ is assumed because of symmetry.

It can be verified in equation (17) that if $Z=0$, the integrand does not tend to zero when $|a| \rightarrow \infty$. The implication is that $G^{W}$ may be singular on the free surface if the source is located on $z=0$. To analyse such behaviour, we write

$$
2 \pi F^{2} G^{W}=2 \pi F^{2}\left(G^{W}-H^{W}\right)+2 \pi F^{2} H^{W}
$$

where

$$
2 \pi F^{2} H^{W}=-\mathrm{i}\left(\int_{-\infty}^{a^{-}}-\int_{a^{+}}^{\infty}\right)\left(\mathscr{E}^{+}+\mathscr{E}^{-}\right) \mathrm{d} a .
$$

It can be shown that $2 \pi F^{2}\left(G^{W}-H^{W}\right)$ is finite. In fact, taking $\int_{a^{+}}^{\infty}(c / b-1) \mathscr{E}^{+} \mathrm{d} a$ as an example, we have

$$
\left|\left(\frac{c}{b}-1\right) \mathscr{E}^{+}\right| \leqslant\left|\frac{c-b}{b}\right|=\frac{c^{2}-b^{2}}{b(c+b)}=\frac{a^{2}-2 \tau a-\tau^{2}}{b(c+b)} .
$$

The last term of the above equation clearly shows that it decays at a rate proportional to $1 / a^{2}$ since $b \rightarrow c=a^{2}$ for $|a| \rightarrow \infty$ as can be seen from (14), which means that the result of the integration is finite. Therefore, we need consider only the last term of equation (18).

We further write

$$
2 \pi F^{2} H^{W}=2 \pi F^{2}\left(H^{W}-\mathscr{G}_{0}^{W}-\mathscr{G}_{1}^{W}\right)+2 \pi F^{2} \mathscr{G}_{0}^{W}+2 \pi F^{2} \mathscr{G}_{1}^{W}
$$

where

$$
2 \pi F^{2} \mathscr{G}_{0}^{W}=-\mathrm{i}\left(\int_{-\infty}^{a^{-}}-\int_{a^{+}}^{\infty}\right)\left(E^{+}+E^{-}\right) \mathrm{d} a,
$$




$$
2 \pi F^{2} \mathscr{G}_{1}^{W}=\tau Y\left(\int_{-\infty}^{a^{-}}-\int_{a^{+}}^{\infty}\right)\left(E^{+}-E^{-}\right) / a \mathrm{~d} a,
$$

and

$$
E^{ \pm}=\exp \left[-\left(-Z+\mathrm{i} Y^{ \pm}\right) a^{2}-\mathrm{i} X a-\mathrm{i}\left(\tau X-Y^{ \pm} / 2\right)\right] .
$$

To show the term in the brackets on the right-hand side of equation (20) is finite, we consider

$$
\int_{a^{+}}^{\infty}\left(\mathscr{E}^{+}-E^{+}-\mathrm{i} \tau Y E^{+} / a\right) \mathrm{d} a
$$

as an example. Using the definitions of $\mathscr{E}^{+}$and $E^{+}$, the integrand of the above equation becomes

$$
\begin{aligned}
\left|\mathscr{E}^{+}-E^{+}-\mathrm{i} \tau Y E^{+} / a\right| & =\left|E^{+}\left\{\exp \left[-\mathrm{i} Y\left(b-a^{2}+1 / 2\right)\right]-1-\mathrm{i} \tau Y / a\right\}\right| \\
& \leqslant\left|\exp \left[-\mathrm{i} Y\left(b-a^{2}+1 / 2\right)\right]-1-\mathrm{i} \tau Y / a\right| .
\end{aligned}
$$

We now consider the limit

$$
\begin{aligned}
L & =\lim _{a \rightarrow \infty} \frac{\exp \left[-\mathrm{i} Y\left(b-a^{2}+1 / 2\right)\right]-1-\mathrm{i} \tau Y / a}{1 / a^{2}} \\
& =\lim _{a \rightarrow \infty} \frac{\exp \left[-\mathrm{i} Y\left(-2 \tau a-\tau^{2}-1 / 4\right) /\left(b+a^{2}-1 / 2\right)\right]-1-\mathrm{i} \tau Y / a}{1 / a^{2}} \\
& =\lim _{a \rightarrow \infty} \frac{\exp (\mathrm{i} \tau Y / a)-1-\mathrm{i} \tau Y / a}{1 / a^{2}} \\
& =\lim _{s \rightarrow 0} \frac{\exp (\mathrm{i} \tau Y s)-1-\mathrm{i} \tau Y s}{s^{2}} \quad \text { with } \quad s=1 / a \\
& =-(\tau Y)^{2} / 2 .
\end{aligned}
$$

Thus the left-hand side of equation (25) decays to zero at a rate not less than $1 / a^{2}$, which means equation (24) in finite. Therefore the analysis can be focused on equations (21) and (22) only.

By using (23) in (21), $\mathscr{G}_{0}^{W}$ can be written

$$
2 \pi F^{2} \mathscr{G}_{0}^{W}=-\mathrm{ie}^{-\mathrm{i} \tau X}\left(\int_{-\infty}^{a^{-}}-\int_{a^{+}}^{\infty}\right)\left[\mathrm{e}^{-(-Z+\mathrm{i} Y) a^{2}-\mathrm{i} X a+\mathrm{i} Y / 2}+\mathrm{e}^{-(-Z-\mathrm{i} Y) a^{2}-\mathrm{i} X a-\mathrm{i} Y / 2}\right] \mathrm{d} a .
$$

The integration limits $\left(-\infty, a^{-}\right]$can be converted into $\left[-a^{-}, \infty\right)$ by changing the integral variable and so $\widetilde{\mathscr{G}_{0}^{W}}$ can be expressed as

$$
2 \pi F^{2} \mathscr{G}_{0}^{W}=-\mathrm{i} \mathrm{e}^{-\mathrm{i} \tau X}\left(I_{0}^{1}+I_{0}^{2}-I_{0}^{3}-I_{0}^{4}\right)
$$

where

$$
\begin{aligned}
& I_{0}^{1}=I_{0}\left(-Z+\mathrm{i} Y,-\mathrm{i} X / 2,-\mathrm{i} Y / 2,-a^{-}\right), \\
& I_{0}^{2}=I_{0}\left(-Z-\mathrm{i} Y,-\mathrm{i} X / 2,+\mathrm{i} Y / 2,-a^{-}\right), \\
& I_{0}^{3}=I_{0}\left(-Z+\mathrm{i} Y,+\mathrm{i} X / 2,-\mathrm{i} Y / 2,+a^{+}\right), \\
& I_{0}^{4}=I_{0}\left(-Z-\mathrm{i} Y,+\mathrm{i} X / 2,+\mathrm{i} Y / 2,+a^{+}\right),
\end{aligned}
$$

and the function $I_{0}\left(p, q, r, t_{0}\right)$ is defined by (B $\left.1 a\right)$ in Appendix B, with its asymptotic properties at large values of $|w|=\left|\left(p t_{0}+q\right) / \sqrt{p}\right|$ being given by (B2), depending on 
$|\arg (w)|$. For a finite value of $q \neq 0$, it can be written that

$$
\arg \left[\left(p t_{0}+q\right) / \sqrt{p}\right]=\arg (q / \sqrt{p})
$$

as $|p| \rightarrow 0$. Thus the phase value of $(q / \sqrt{p})$ is considered in the following asymptotic analysis.

The following variables are introduced:

$$
\mathscr{H}=\sqrt{(-Z)^{2}+Y^{2}} \text { and } \theta=\arctan [Y /(-Z)]
$$

and because only $Y \geqslant 0$ is considered, $0 \leqslant \theta \leqslant \pi / 2$, as $-Z \geqslant 0$. The phase values of $(q / \sqrt{p})$ corresponding to $I_{0}^{n}(n=1, \cdots, 4)$ are respectively

$$
\begin{aligned}
& \pi / 4 \leqslant\left|\arg (q / \sqrt{p})_{I_{0}^{1}}\right| \leqslant \pi / 2, \quad \pi / 2 \leqslant\left|\arg (q / \sqrt{p})_{I_{0}^{2}}\right| \leqslant 3 \pi / 4, \\
& \pi / 2 \leqslant\left|\arg (q / \sqrt{p})_{I_{0}^{3}}\right| \leqslant 3 \pi / 4, \quad \pi / 4 \leqslant\left|\arg (q / \sqrt{p})_{I_{0}^{4}}\right| \leqslant \pi / 2,
\end{aligned}
$$

noting that $X<0$ has been assumed. Here we consider the case when $|q / \sqrt{p}| \rightarrow \infty$ based on Appendix B, and the asymptotic expansion will be dominated by the terms with phase values of $(q / \sqrt{p})$ larger than $\pi / 2$. This means that only $I_{0}^{2}$ and $I_{0}^{3}$ have to be retained here. Thus

$$
\mathscr{G}_{0}^{W} \approx \widetilde{\mathscr{G}_{0}^{W}}
$$

where

$$
\widetilde{\mathscr{G}_{0}^{W}}=\frac{-\mathrm{i} \mathrm{e}^{-\mathrm{i} \tau X}}{2 \pi F^{2}}\left(\sqrt{\frac{\pi}{-Z-\mathrm{i} Y}} \mathrm{e}^{-X^{2} /[4(-Z-\mathrm{i} Y)]-\mathrm{i} Y / 2}-\sqrt{\frac{\pi}{-Z+\mathrm{i} Y}} \mathrm{e}^{-X^{2} /[4(-Z+\mathrm{i} Y)]+\mathrm{i} Y / 2}\right),
$$

which can be rearranged by using (28)

$$
\widetilde{\mathscr{G}_{0}^{W}}=\frac{\exp \left[Z X^{2} /\left(4 \mathscr{H}^{2}\right)-\mathrm{i} \tau X\right]}{F^{2} \sqrt{\pi \mathscr{H}}} \sin \left[(\theta-Y) / 2-Y X^{2} /\left(4 \mathscr{H}^{2}\right)\right] .
$$

The above expression captures the behaviour of high oscillation with indefinitely increasing amplitude and indefinitely decreasing wavelength, since

$$
\widetilde{\mathscr{G}}(X, Y)=\widetilde{\mathscr{G}_{0}^{W}}(Z=0)=\frac{\mathrm{e}^{-\mathrm{i} \tau X}}{\sqrt{\pi} F^{2}} Y^{-1 / 2} \cos \left(\frac{X^{2}}{4 Y}+\frac{Y}{2}+\frac{\pi}{4}\right) .
$$

On the other hand, when $Z<0$ and $Y \rightarrow 0$, equation (29b) has a finite value. Indeed, the amplitude of $\widetilde{\mathscr{G}_{0}^{W}}$ in $(29 b)$ is given by

$$
\frac{\exp \left[Z X^{2} /\left(4 \mathscr{H}^{2}\right)\right]}{F^{2} \sqrt{\pi \mathscr{H}}}
$$

which is proportional to $O\left(Y^{-1 / 2}\right)$ if $Z=0$, i.e. singular as $Y \rightarrow 0$, and is finite as $Y(<-Z) \rightarrow 0$ if $Z<0$. Thus, the limit is non-uniform and depends on whether $\theta=\pi / 2(Z=0)$ or $\theta<\pi / 2(Z<0)$. Another point here is $\overline{\mathscr{G}_{0}^{W}}$ does not decay with $X$ when $Z=0$ but decays exponentially when $Z<0$.

The oscillatory behaviour in $(29 b)$ is demonstrated through the triangular function with the following variable:

$$
-Y X^{2} /\left(4 \mathscr{H}^{2}\right)
$$

which gives an increasing wavenumber $X^{2} /\left(4 Y^{2}\right)$ as $Y \rightarrow 0$. This means that $\widetilde{\mathscr{G}_{0}^{W}}$ 

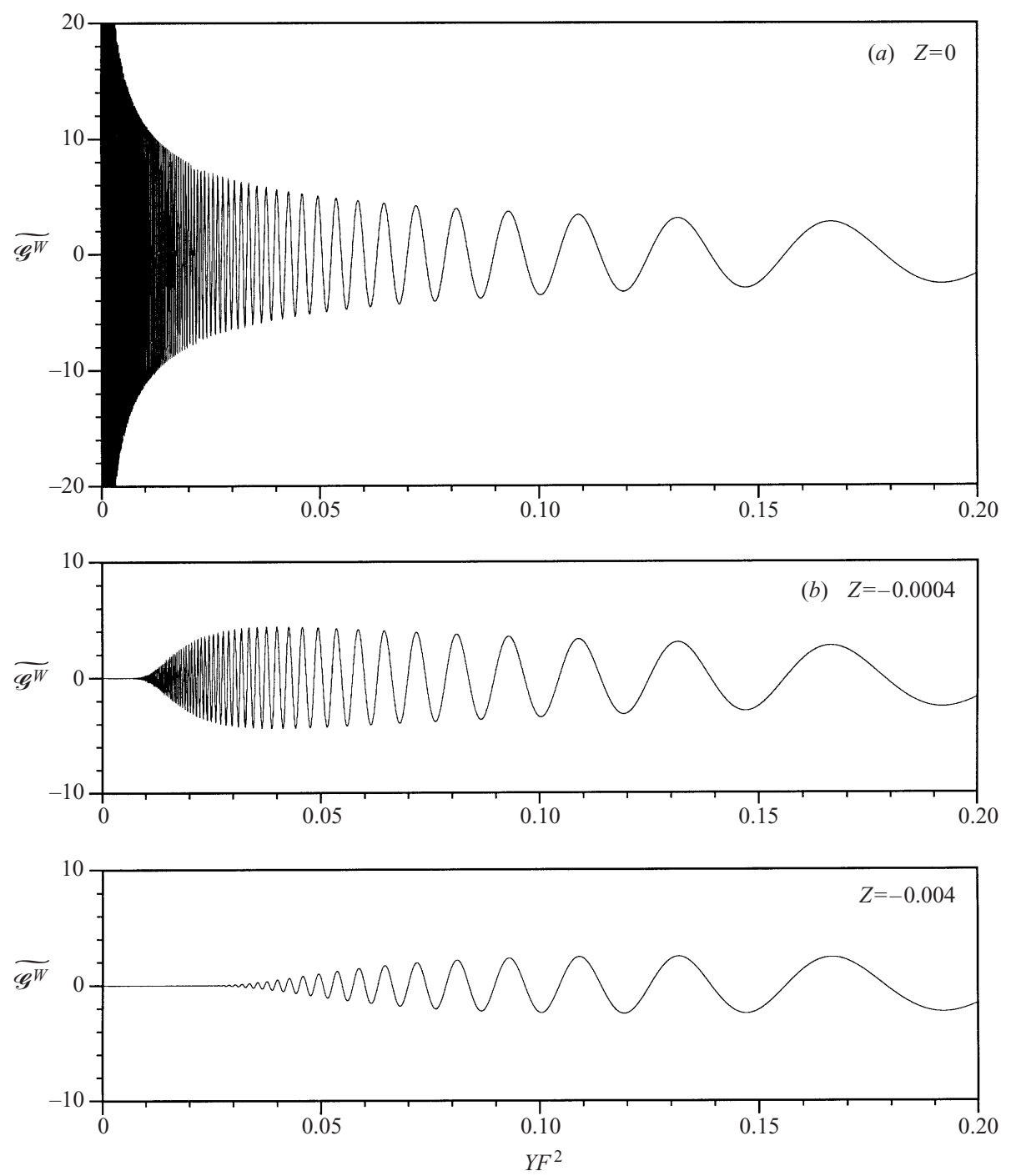

FiguRE 1. (a) Singular and highly oscillatory term of the wave component expressed by (34) at $\tau=0, X=-8$ and $Z=0$. (b) Highly oscillatory term of the wave component given by (34) at $Z=-0.0004$. (c) Highly oscillatory term of the wave component given by (34) at $Z=-0.004$.

oscillates more and more rapidly as $Y \rightarrow 0$, i.e. when a field point tends to the track of the source point located close to or at the free surface. This singular (when $Z=0$ ) and highly oscillatory behaviour of $\widetilde{\mathscr{G}_{0}^{W}}$ is shown graphically in figure 1 which depicts $\widetilde{\mathscr{G}_{0}^{W}}(Y)$ at $Z=0$ (figure $1 a$ ), $Z=-0.0004$ (figure $1 b$ ) and $Z=-0.004$ (figure $1 c$ ), when $X=-8, F=1 / 2$ and $\tau=0$ (see below for $\tau \neq 0$ ).

Another interesting feature of the asymptotic expression $(29 b)$ is that the result at $\tau \neq 0$ can be obtained by multiplying the result at $\tau=0$ with

$$
\exp (-\mathrm{i} \tau X) \text {. }
$$

This remarkable result is due to the fact that the parabola $b+1 / 2=a^{2}$ is the leading term of the asymptotic expansion for open dispersion curves (see equations 
(14) and (25)) and $a=F^{2} \alpha-\tau$ represents a simple shift $(\tau, 0)$ of the plane $F^{2}(\alpha, \beta)$. A shift of the origin of the Fourier plane to $\left(\alpha_{0}, \beta_{0}\right)$ yields an oscillatory factor of type $\exp \left[-\mathrm{i}\left(\alpha_{0} x+\beta_{0} y\right)\right]$.

In a similar way, the component $\mathscr{G}_{1}^{W}$ defined by (22) can be written as

$$
2 \pi F^{2} \mathscr{G}_{1}^{W}=\tau Y \mathrm{e}^{-\mathrm{i} \tau X}\left(\int_{-\infty}^{a^{-}}-\int_{a^{+}}^{\infty}\right)\left[\mathrm{e}^{-(-Z+\mathrm{i} Y) a^{2}-\mathrm{i} X a+\mathrm{i} Y / 2}-\mathrm{e}^{-(-Z-\mathrm{i} Y) a^{2}-\mathrm{i} X a-\mathrm{i} Y / 2}\right] / a \mathrm{~d} a,
$$

which can again be expressed as

$$
2 \pi F^{2} \mathscr{G}_{1}^{W}=\tau Y \mathrm{e}^{-\mathrm{i} \tau X}\left(-I_{1}^{1}+I_{1}^{2}-I_{1}^{3}+I_{1}^{4}\right)
$$

with

$$
\begin{aligned}
& I_{1}^{1}=I_{1}\left(-Z+\mathrm{i} Y,-\mathrm{i} X / 2,-\mathrm{i} Y / 2,-a^{-}\right), \\
& I_{1}^{2}=I_{1}\left(-Z-\mathrm{i} Y,-\mathrm{i} X / 2,+\mathrm{i} Y / 2,-a^{-}\right), \\
& I_{1}^{3}=I_{1}\left(-Z+\mathrm{i} Y,+\mathrm{i} X / 2,-\mathrm{i} Y / 2,+a^{+}\right), \\
& I_{1}^{4}=I_{1}\left(-Z-\mathrm{i} Y,+\mathrm{i} X / 2,+\mathrm{i} Y / 2,+a^{+}\right),
\end{aligned}
$$

where the function $I_{1}\left(p, q, r, t_{0}\right)$ is defined by (B $\left.3 a\right)$ in Appendix B, and its asymptotic properties are given by (B 10), in terms of $\arg (q / \sqrt{p})$. The phase values of $(q / \sqrt{p})$ for $I_{1}^{n}(n=1, \ldots, 4)$ are

$$
\begin{aligned}
& \pi / 4 \leqslant\left|\arg (q / \sqrt{p})_{I_{1}^{1}}\right| \leqslant \pi / 2, \quad \pi / 2 \leqslant\left|\arg (q / \sqrt{p})_{I_{1}^{2}}\right| \leqslant 3 \pi / 4, \\
& \pi / 2 \leqslant\left|\arg (q / \sqrt{p})_{I_{1}^{3}}\right| \leqslant 3 \pi / 4, \quad \pi / 4 \leqslant\left|\arg (q / \sqrt{p})_{I_{1}^{4}}\right| \leqslant \pi / 2 .
\end{aligned}
$$

Applying equation (B 10) in the Appendix B to (32a), we can see that the term of the exponential integral will be of $O(Y)$ when $Y \rightarrow 0$ due to cancellation. The major contribution is then from $I_{1}^{2}$ and $I_{1}^{3}$ which have phase values larger than $\pi / 2$ and are of $O(\sqrt{Y})$. Therefore, $\mathscr{G}_{1}^{W} \approx \overline{\mathscr{G}_{1}^{W}}$ where

$$
\widetilde{\mathscr{G}_{1}^{W}}=\frac{\tau Y \mathrm{e}^{-\mathrm{i} \tau X}}{2 \sqrt{\pi} F^{2}}\left(\frac{\sqrt{-Z-\mathrm{i} Y}}{\mathrm{i} X / 2} \mathrm{e}^{-X^{2} /[4(-Z-\mathrm{i} Y)]-\mathrm{i} Y / 2}+\frac{\sqrt{-Z+\mathrm{i} Y}}{\mathrm{i} X / 2} \mathrm{e}^{-X^{2} /[4(-Z+\mathrm{i} Y)]+\mathrm{i} Y / 2}\right),
$$

which can be rearranged by using (28)

$$
\widetilde{\mathscr{G}_{1}^{W}}=-i 2 \tau \frac{\exp \left[Z X^{2} /\left(4 \mathscr{H}^{2}\right)-\mathrm{i} \tau X\right]}{F^{2} \sqrt{\pi / \mathscr{H}}(X / Y)} \cos \left[(\theta+Y) / 2+Y X^{2} /\left(4 \mathscr{H}^{2}\right)\right] .
$$

This equation shows that $\widetilde{\mathscr{G}_{1}^{W}}$ is also highly oscillatory due to the triangular function of variable $Y X^{2} /\left(4 \mathscr{H}^{2}\right)$ as $\mathscr{H} \rightarrow 0$, similar to $\widetilde{\mathscr{G}_{0}^{W}}$ in $(29 b)$. The difference is that $\overline{\mathscr{G}_{1}^{W}}$ is not singular and it goes to zero at a rate proportional to $Y^{3 / 2}$.

Therefore, the far-field wave component $\mathscr{G}^{W}$ can be approximated by

$$
\mathscr{G}^{W} \approx \widetilde{\mathscr{G}^{W}}
$$

as $Y \rightarrow 0$, where the highly-oscillatory and singular term $\widetilde{\mathscr{G}^{W}}$ is defined by

$$
\widetilde{\mathscr{G}^{W}}=\widetilde{\mathscr{G}_{0}^{W}}+\widetilde{\mathscr{G}_{1}^{W}} \text {. }
$$


By using (29b) and (33b), this becomes

$\widetilde{\mathscr{G}^{W}}=\mathrm{e}^{Z X^{2} /\left(4 \mathscr{H}^{2}\right)-\mathrm{i} \tau X}\left(\frac{\sin \left[(\theta-Y) / 2-Y X^{2} /\left(4 \mathscr{H}^{2}\right)\right]}{F^{2} \sqrt{\pi \mathscr{H}}}-\mathrm{i} 2 \tau \frac{\cos \left[(\theta+Y) / 2+Y X^{2} /\left(4 \mathscr{H}^{2}\right)\right]}{F^{2} \sqrt{\pi / \mathscr{H}}(X / Y)}\right)$.

It is interesting to see that equation (34) reduces to the following form:

$$
\widetilde{\mathscr{G}^{W}}=\frac{\exp \left[Z X^{2} /\left(4 \mathscr{H}^{2}\right)\right]}{F^{2} \sqrt{\pi \mathscr{H}}} \sin \left[(\theta-Y) / 2-Y X^{2} /\left(4 \mathscr{H}^{2}\right)\right]
$$

when $\tau=0$. This result may be compared with that of Ursell $(1960,1988)$ for the steady Neumann-Kelvin problem. It can be found that the sine function is in full agreement. There is a difference in the amplitude, but this is because Ursell's result is for a moving pressure while equation (35) is for a moving source. When the relationship between moving pressure and a moving source is used, the result here is identical to Ursell's.

The behaviour of (34) is shown by figure 2 which depicts the real part $(a)$ and imaginary part $(b)$ of the wave component $\mathscr{G}^{W}$ for $\tau=1 / 2=F, X=-20$ and $Z=-0.002$. The thin solid lines represent the wave component $\mathscr{G}^{W}$ evaluated by using the single integral in equation (7) along the corresponding open dispersion curves. Extremely rapid oscillation of $\mathscr{G}^{W}$ with large amplitude is evident, as expected from the above analysis. The difference between the wave component $\mathscr{G}^{W}$ and the first leading term of asymptotic expansion (34), i.e.

$$
\mathscr{G}^{W}-\widetilde{\mathscr{G}_{0}^{W}},
$$

is represented by the thick solid lines in figure 2. It can be seen that $\mathscr{G}^{W}-\widetilde{\mathscr{G}_{0}^{W}}$ has much smaller amplitude. The dashed lines in figure 2 represent the difference between the wave component $\mathscr{G}^{W}$ and its asymptotic expansion $\mathscr{G}^{W}$ given by (34), i.e.

$$
\mathscr{G}^{W}-\widetilde{\mathscr{G}^{W}}=\mathscr{G}^{W}-\left(\widetilde{\mathscr{G}_{0}^{W}}+\widetilde{\mathscr{G}_{1}^{W}}\right) .
$$

The result shows that the oscillation of $\mathscr{G}^{W}-\widetilde{\mathscr{G}^{W}}$ is hardly noticeable. This means that $\frac{\mathscr{G}^{W}}{\text { is an }}$ axcellent approximation for capturing the singular and highly-oscillatory behaviour of $\mathscr{G}^{W}$.

\section{Discussion and conclusions}

The above analysis is based on the asymptotic behaviour of the complex error function $\operatorname{Cef}(w)$ given in Appendix A as $|w|=\left|\left(p t_{0}+q\right) / \sqrt{p}\right| \rightarrow \infty ; p, q$ and $t_{0}$ are related to variables of $I_{0}^{n}$ and $I_{1}^{n}(n=1, \ldots, 4)$ in equations $(26 b)$ and $(32 b)$ respectively. Thus it is essential to assume $X \neq 0$ if $Y \rightarrow 0$ gives $|w| \rightarrow \infty$. This means that the result obtained here is valid only when the field point is near the source track but not close to the source.

The wave component $\mathscr{G}^{W}$ is defined by (7) through a single integral along closed and open dispersion curves. The wave system associated with a closed dispersion curve is not singular as the integrand is finite. The wave systems associated with open dispersion curves can be singular and highly oscillatory, depending on the position of the field and source points. The singular and highly-oscillatory behaviour can be captured by $\mathscr{G}_{0}^{W}$ and $\mathscr{G}_{1}^{W}$ in equations (21) and (22). The remaining part of the wave component may be oscillatory under the same condition but its amplitude tends to 

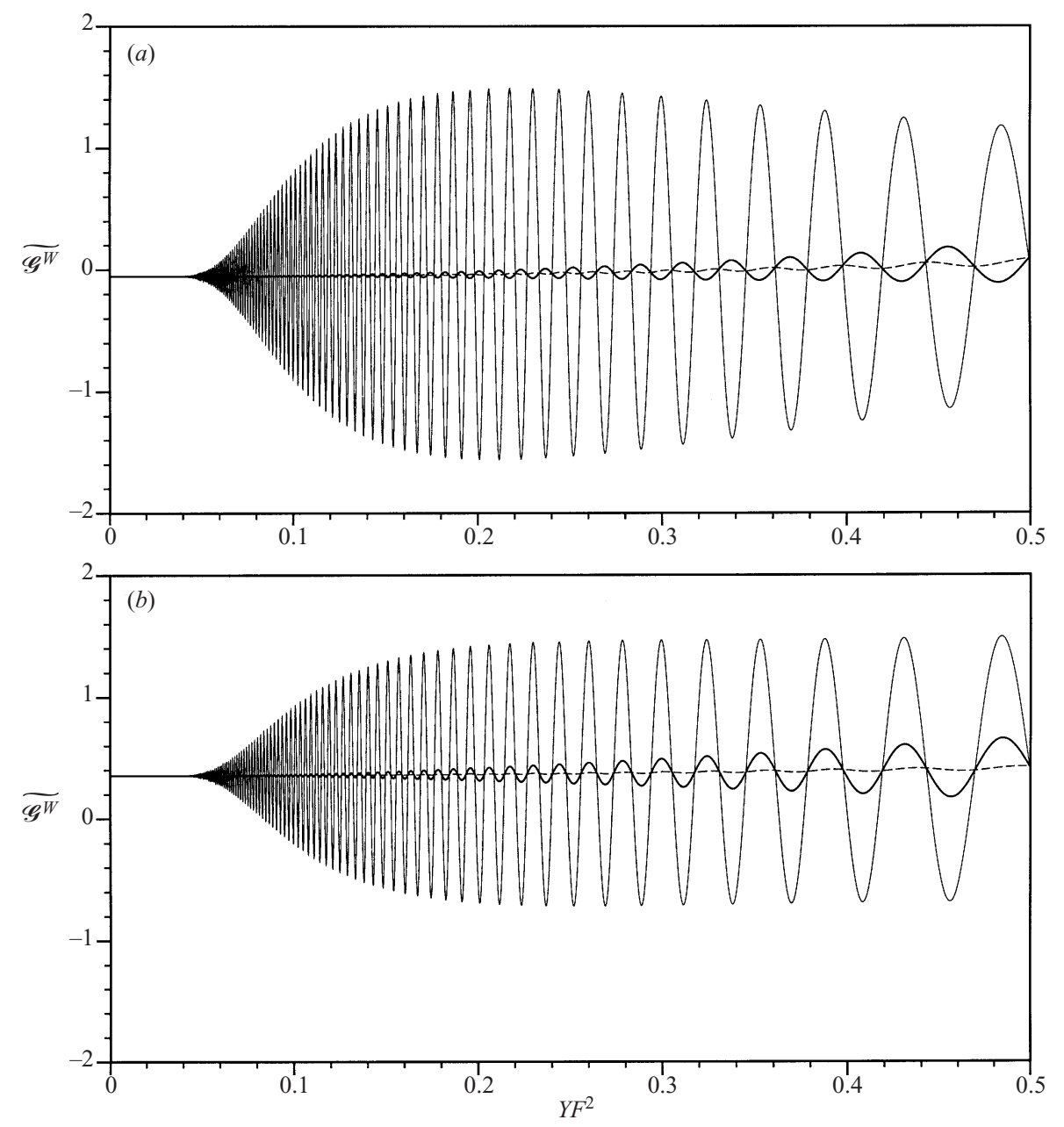

FIGURE 2. Real part $(a)$ and imaginary part $(b)$ of the wave component. The parameters of the computation are $\tau=1 / 2=F, Z=-0.002$ and $X=-20$. The thin lines represent the wave component obtained by the single integral (7) associated with the open dispersion curves. The thicker lines represent the values of the wave component after subtraction of the first term in (34). The dashed curves show the values of the wave component after subtraction of both terms of (34) - the asymptotic expansions of the wave component associated with the open dispersion curves.

zero. Therefore, it is far less problematic in the numerical computation than $\mathscr{G}_{0}^{W}$ and $\mathscr{G}_{1}^{W}$.

The complex nature of the wave component captured by $\mathscr{G}_{0}^{W}$ gives insight into the difficulty in the numerical prediction of the motion of a ship advancing in waves. A commonly used method is to convert the Laplace equation in the fluid domain into an integral equation over the boundary of the fluid domain, by using the Green function. The integration over the free surface is further transformed into two line integrals: one at infinity and another along the waterline of the ship (Brard 1972). The former is often ignored in the analysis. Such a practice may have to be justified, especially when both source and field points are on the free surface. Even when the velocity potential can be represented by a source distribution over the body surface 
and along the waterline, a stable numerical solution is still not easy to achieve because of difficulty when calculating the Green function.

An alternative to this Green function method is the Rankine source method (Nakos \& Sclavounos 1990). It avoids the difficulty of evaluating a function like $G^{W}$ in equation (7) as the Green function used now is simply $1 / r$. But as the sources are distributed over the free surface, only those wave components with wavelength much larger than the panel size can be modelled in the analysis. Also it is quite uncertain what the radiation condition is and how it should be imposed if the wave feature described in this paper is to be captured.

It should be noticed that the Green function given in equations (1)-(3) is based on the linear theory. One of the assumptions used in this theory is that the wave height is much smaller than the wavelength. Such a condition is clearly not satisfied by $G$ when the source is located near or on the free surface. Therefore, it may be argued that the feature shown through $\widetilde{\mathscr{G}_{0}^{W}}$ in equation $(29 a)$ is a result of linearization. When the nonlinear term is included, the wave feature may be quite different. Even based on the framework of the linear theory, the wave behind a ship is due to some continuous distribution of sources, which may give a wave structure different from that due to a single source. It is expected that the results of the present study will help to resolve these difficult issues.

The authors are grateful to Professor F. Ursell for his most valuable comments on an early version of the paper.

\section{Appendix A. Complex error function}

The complex error function is defined by

$$
\operatorname{Cef}(w)=w \mathrm{e}^{w^{2}} \int_{w}^{\infty} \mathrm{e}^{-t^{2}} \mathrm{~d} t=w \mathrm{e}^{w^{2}} \frac{\sqrt{\pi}}{2}[1-\operatorname{erf}(w)]
$$

where $\operatorname{erf}(w)$ is the usual error function defined by (7.1.1) in Abramowitz \& Stegun (1964). It follows from (7.1.5) in Abramowitz \& Stegun (1964) that Cef(w) can be expressed by a series expansion

$$
\operatorname{Cef}(w)=w \mathrm{e}^{w^{2}}\left(\sqrt{\pi} / 2-\sum_{n=0}^{\infty} \frac{(-1)^{n} w^{2 n+1}}{n !(2 n+1)}\right)
$$

valid when $|w|<\infty$. To find an asymptotic expansion of $\operatorname{Cef}(w)$ for large $|w|$, integration by parts is performed repeatedly on equation (A 1). This gives

$$
\operatorname{Cef}(w)=\frac{1}{2}-\frac{1}{2^{2} w^{2}}+\frac{1 \times 3}{2^{3} w^{4}}-\cdots+(-1)^{n} \frac{1 \times 3 \times 5 \cdots(2 n-1)}{2^{n+1} w^{2 n}}+R_{n}(w)
$$

where $R_{n}(w)$ is the remainder after $n$ terms and is defined by

$$
R_{n}(w)=(-1)^{n+1} \frac{1 \times 3 \times 5 \cdots(2 n+1)}{2^{n+1}} w \mathrm{e}^{w^{2}} \int_{w}^{\infty} \frac{\mathrm{e}^{-t^{2}}}{t^{2 n+2}} \mathrm{~d} t .
$$

To estimate $R_{n}(w)$ with $w=u+\mathrm{i} v$, let $|\arg (w)| \leqslant \pi / 2$, and choose the path of integration in (A $4 a$ ) to be the infinite line segment beginning at the point $t=w$ and parallel to the real axis, i.e. $t=T+\mathrm{i} v$ with $u \leqslant T<\infty$ along which

$$
\left|\mathrm{e}^{w^{2}-t^{2}}\right|=\mathrm{e}^{u^{2}-T^{2}} \text { and }|t|^{-(2 n+2)} \leqslant|w|^{-(2 n+2)} .
$$


Therefore

$$
\left|R_{n}(w)\right| \leqslant \frac{1 \times 3 \times 5 \cdots(2 n+1)}{2^{n+1}|w|^{2 n+1}} \int_{u}^{\infty} \mathrm{e}^{u^{2}-T^{2}} \mathrm{~d} T \leqslant \sqrt{\pi} \frac{1 \times 3 \times 5 \cdots(2 n+1)}{2^{n+2}|w|^{2 n+1}}
$$

where the relation

$$
\int_{u}^{\infty} \mathrm{e}^{u^{2}-T^{2}} \mathrm{~d} T=\frac{\sqrt{\pi}}{2} \mathrm{e}^{u^{2}}[1-\operatorname{erf}(u)] \leqslant \sqrt{\pi} / 2
$$

has been used. Thus as $|w| \rightarrow \infty$ the product $w^{2 n} R_{n}(w)$ converges uniformly to zero if $|\arg (w)| \leqslant \pi / 2$. Under this condition, the asymptotic expansion of $\operatorname{Cef}(w)$ may be written as

$$
\operatorname{Cef}(w)=\frac{1}{2}+\sum_{n=1}^{\infty}(-1)^{n} \frac{1 \times 3 \times 5 \cdots(2 n-1)}{2\left(2 w^{2}\right)^{n}}
$$

whose error is less than the value estimated by (A $4 b)$.

For $|\arg (w)|>\pi / 2$, the identity

$$
\operatorname{Cef}(w)=\operatorname{Cef}(-w)+\sqrt{\pi} w \mathrm{e}^{w^{2}}
$$

can be used. Since $|\arg (-w)|<\pi / 2$, (A 6) is applicable to Cef $(-w)$. The above equation (A 7) can also be verified directly by using the series expansion in (A 2).

In brief, the limit value of $\operatorname{Cef}(w)$ as $|w| \rightarrow \infty$ can be expressed as

$$
\lim _{|w| \rightarrow \infty} \operatorname{Cef}(w)=\left\{\begin{array}{ll}
1 / 2+O\left(w^{-2}\right), & |\arg (w)| \leqslant \pi / 2 \\
1 / 2+\sqrt{\pi} w e^{w^{2}}+O\left(w^{-2}\right), & |\arg (w)|>\pi / 2
\end{array}\right\}
$$

Note that the limit of $\operatorname{Cef}(w)$ at $|w| \rightarrow \infty$ is continuous for $|\arg (w)|=\pi / 2$, as $\sqrt{\pi} w \mathrm{e}^{w^{2}}$ tends to zero at $|w| \rightarrow \infty$ if $|\arg (w)=\pi / 2|$.

\section{Appendix B. Integrals related to the complex error function}

One elementary integral related to the above complex error function is given in (7.4.32) of Abramowitz \& Stegun (1967) and expressed here by using $\operatorname{Cef}(w)$ given in Appendix A

$$
\begin{aligned}
I_{0}\left(p, q, r, t_{0}\right) & =\int_{t_{0}}^{\infty} \mathrm{e}^{-\left(p t^{2}+2 q t+r\right)} \mathrm{d} t=\mathrm{e}^{-\left(r-q^{2} / p\right)} \int_{t_{0}}^{\infty} \mathrm{e}^{-(p t+q)^{2} / p} \mathrm{~d} t \\
& =\frac{\mathrm{e}^{-\left(p t_{0}^{2}+2 q t_{0}+r\right)}}{p t_{0}+q}\left(\frac{p t_{0}+q}{\sqrt{p}} \mathrm{e}^{\left(p t_{0}+q\right)^{2} / p} \int_{\left(p t_{0}+q\right) / \sqrt{p}}^{\infty} \mathrm{e}^{-u^{2}} \mathrm{~d} u\right) \\
& =\frac{\mathrm{e}^{-\left(p t_{0}^{2}+2 q t_{0}+r\right)}}{p t_{0}+q} \operatorname{Cef}\left[\left(p t_{0}+q\right) / \sqrt{p}\right] .
\end{aligned}
$$

When $|w|=\left|\left(p t_{0}+q\right) / \sqrt{p}\right| \rightarrow \infty$, equation (A 8) leads to

$$
I_{0}\left(p, q, r, t_{0}\right)= \begin{cases}\frac{1}{2} \mathrm{e}^{-\left(p t_{0}^{2}+2 q t_{0}+r\right)} /\left(p t_{0}+q\right), & |\arg (w)| \leqslant \pi / 2 \\ \frac{1}{2} \mathrm{e}^{-\left(p t_{0}^{2}+2 q t_{0}+r\right)} /\left(p t_{0}+q\right)+(\sqrt{\pi / p}) \mathrm{e}^{q^{2} / p-r}, & |\arg (w)|>\pi / 2 .\end{cases}
$$

The second term on the right-hand side of (B 2) is of form $O\left(\mathrm{e}^{q^{2} / p} / \sqrt{p}\right)$ as $|q / \sqrt{p}| \rightarrow \infty$ and independent of the value of the lower limit $t_{0}$ of the integral. 
Another integral involved in the asymptotic analysis in this paper is of the form

$$
I_{1}\left(p, q, r, t_{0}\right)=\int_{t_{0}}^{\infty} \mathrm{e}^{-\left(p t^{2}+2 q t+r\right)} / t \mathrm{~d} t
$$

which can be rewritten as

$$
I_{1}\left(p, q, r, t_{0}\right)=\int_{0}^{p} F(u) \mathrm{d} u+I_{1}\left(0, q, r, t_{0}\right)
$$

in which the path of integration may be taken as a straight line from 0 to $p$, and

$$
F(u)=\partial I_{1}\left(u, q, r, t_{0}\right) / \partial u=-\int_{t_{0}}^{\infty} t \mathrm{e}^{-\left(u t^{2}+2 q t+r\right)} \mathrm{d} t=-\frac{\mathrm{e}^{-\left(u t^{2}+2 q t+r\right)}}{2 u}+\left(\frac{q}{u}\right) I_{0}\left(u, q, r, t_{0}\right) .
$$

Our main interest here is to analyse $I_{1}\left(p, q, r, t_{0}\right)$ when $p \rightarrow 0$ which gives $u \rightarrow 0$. From equation (B 2), it can be seen that the two terms of the right-hand side of (B 4) cancel each other when $\left|\arg \left[\left(u t_{0}+q\right) / \sqrt{u}\right]\right| \leqslant \pi / 2$. For $\left|\arg \left[\left(u t_{0}+q\right) / \sqrt{u}\right]\right|>\pi / 2$, equation (B 2) leads to

$$
F(u)=\left(\frac{q}{u}\right) \sqrt{\pi} \mathrm{e}^{-r} \frac{\exp \left(q^{2} / u\right)}{\sqrt{u}} .
$$

Thus, we may estimate the first term on the right-hand side of (B $3 b)$ as

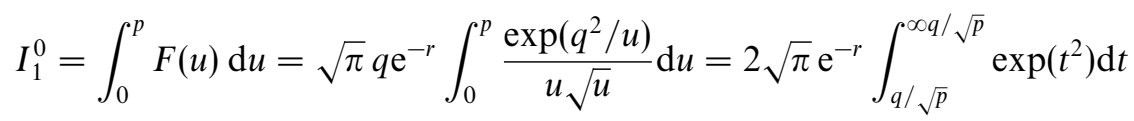

where we have changed the integral variable $u=(q / t)^{2}$. Furthermore, we are interested only in the case when $\pi / 2 \leqslant \arg (q / \sqrt{p}) \leqslant 3 \pi / 4$ and $-3 \pi / 4 \leqslant \arg (q / \sqrt{p}) \leqslant-\pi / 2$ because of $I_{1}^{n}(n=1, \ldots, 4)$ in equation $(32 b)$. If we use $v=\mp i t$ for these two cases respectively, equation (7.2.14) of Abramowitz \& Stegun gives

$$
I_{1}^{0}=\pi \mathrm{e}^{-r}( \pm \mathrm{i}) \operatorname{erfc}(\mp \mathrm{i} q / \sqrt{p})
$$

Subsequently equation (7.2.14) of Abramowitz \& Stegun gives

$$
I_{1}^{0} \approx-(\sqrt{\pi p} / q) \exp \left(q^{2} / p-r\right) .
$$

The second term on the right-hand side of (B $3 b)$ is evaluated by

$$
I_{1}\left(0, q, r, t_{0}\right)=\mathrm{e}^{-r} \int_{t_{0}}^{\infty} \mathrm{e}^{-2 q t} / t \mathrm{~d} t=\mathrm{e}^{-r} \int_{1}^{\infty} \mathrm{e}^{-2 q t_{0} u} / u \mathrm{~d} u=\mathrm{e}^{-r} E_{1}\left(2 q t_{0}\right)
$$

in which $E_{1}(w)$ is the exponential integral function defined by (5.1.4) in Abramowitz $\&$ Stegun. Thus

$$
I_{1}\left(p, q, r, t_{0}\right)= \begin{cases}\mathrm{e}^{-r} E_{1}\left(2 q t_{0}\right), & \left|\arg \left[\left(p t_{0}+q\right) / \sqrt{p}\right]\right| \leqslant \pi / 2 \\ \mathrm{e}^{-r} E_{1}\left(2 q t_{0}\right)-(\sqrt{\pi p} / q) \exp \left(q^{2} / p-r\right), & \left|\arg \left[\left(p t_{0}+q\right) / \sqrt{p}\right]\right|>\pi / 2 .\end{cases}
$$

\section{REFERENCES}

Abramowitz, M. \& Stegun, I. A. 1964 Handbook of Mathematical Functions. Dover.

BRARD, R. 1972 The representation of a given ship form by a singularity distribution when the boundary condition on the free surface is linearized. J. Ship Res. 16, 79-92. 
Chen, X. B. \& Noblesse, F. 1997 Dispersion relation and far-field waves. In Proc. 12th Intl Workshop on Water Waves and Floating Bodies, Carry-Le-Rouet, France, pp. 31-35.

Clarisse, J. M. \& Newman, J. N. 1994 Evaluation of the wave-resistance Green function: Part 3 The single integral near the singular axis. J. Ship Res. 38, 1-8.

Euvrard, D. 1983 Les mille et une faceties de la fonction de Green du problème de la résistance de vagues. Rapport de Recherche, ENSTA No. 144.

Lamb, H. 1932 Hydrodynamics, 6th Edn. Cambridge University Press.

Lighthill, J. 1978 Wave in Fluids. Cambridge University Press.

Nakos, D. E. \& Sclavounos, P. D. 1990 On steady and unsteady ship wave patterns. J. Fluid Mech. 215, 263-288.

Noblesse, F. \& Chen, X. B. 1995 Decomposition of free-surface effects into wave and near-field components. Ship Technol. Res. 42, 167-185.

Noblesse, F. \& YANG, C. 1995 Fourier-Kochin Formulation of wave diffraction-radiation by ships or offshore structures. Ship Technol. Res. 42, 115-139.

Peters, A. S. 1949 A new treatment of the ship wave problem. Commun. Pure Appl. Maths 2, $123-148$

Ursell, F. 1960 On Kelvin's ship-wave pattern. J. Fluid Mech. 8, 418-431.

URSELL, F. 1988 On the theory of the Kelvin ship-wave source: asymptotic expansion of an integral. Proc. R. Soc. Lond. A 418, 81-93.

Wehausen, J. V. \& Laitone, E. V. 1960 Surface waves. In Handbach des Physik (ed. S. Flugg), vol. 9, pp. 446-778. Springer. 